\title{
Interaction Studies of Master Cardiac Transcription Factors NKX2.5 and TBX5
}

Saai Suryanarayanan* Shayan Ashur*, Shichang Li and Hyun-Joo Nam

*equal contribution

Department of Bioengineering, University of Texas at Dallas

Master cardiac transcription factors (TFs), such as NKX2.5 and TBX5, play an important role in cardiac morphogenesis. Mutations in NKX2.5 and TBX5 are associated with congenital heart diseases (CHDs) such as atrial and ventral septal defects, atrio-ventricular block, and other progressive cardiac disorders. NKX2.5 and TBX5 interact and synergistically activate downstream target genes. The crystal structure of NKX2.5/TBX5 complex with the target DNA (ANF-252) showed protein-protein interactions between their DNA binding domains. NKX2.5 and TBX5 interact through evolutionarily conversed residues, Lys158 of NKX2.5, Asp140 of TBX5, and Pro 145 of TBX5. To quantify the interactions among the cardiac transcription factors and the DNA, we performed Isothermal Titration Calorimetry (ITC) experiments using the DNA binding domains of wild type NKX2.5 and NKX2.5 K158A mutant with TBX5. The data showed that the presence of TBX5 does not affect the DNA binding affinity of the wild type NKX2.5. The NKX2.5 K158A mutant by itself showed reduced DNA binding compared to the wild type. However, in the presence of TBX5, the binding affinity was restored to the extent of the wild type. Studies presented here will facilitate better understanding of combinational interactions among cardiac transcription factors and help develop potential therapeutics for the associated disease conditions. 